\title{
Sexuelle Dysfunktionen und sexuelle Zufriedenheit bei Patientinnen mit posttraumatischer Belastungsstörung
}

\author{
Angelika Haase Anne Boos Sabine Schönfeld Jürgen Hoyer \\ Klinische Psychologie und Psychotherapie, Technische Universität Dresden, Deutschland
}

\section{Schlüsselwörter}

Posttraumatische Belastungsstörung · Sexuelle

Dysfunktionen - Sexuelle Funktionsstörungen .

Sexuelle Zufriedenheit · Sexualität

\section{Zusammenfassung}

Hintergrund: Sexuelle Dysfunktionen sind ein häufiges Problem bei Patientinnen mit posttraumatischer Belastungsstörung (PTB). Kaum untersucht ist, ob ein Zusammenhang zwischen der Art des Traumas (sexuell vs. nichtsexuell) und der Häufigkeit sexueller Dysfunktionen bzw. Zufriedenheit besteht und welche Rolle komorbide Depressionen dabei spielen. Zudem wurden verschiedene Störungsbilder (PTB, Angst, Depression) in Bezug auf sexuelle Funktions-/Zufriedenheitsbeeinträchtigungen vergleichend untersucht (klinische Spezifität). Patientinnen und Methoden: Nach der standardisierten klinischen Diagnostik wurden 351 ambulante Psychotherapiepatientinnen folgenden 3 Gruppen zugeteilt: Patientinnen mit PTB $(n=89)$, mit anderen Angststörungen ( $n=157$ ) und mit depressiven Störungen ( $n=$ 105). Informationen zur sexuellen Zufriedenheit und Funktionsfähigkeit wurden mit dem Kurzfragebogen für Sexualität (KFS) erhoben. Die Art des Traumas (sexuell vs. nichtsexuell) wurde per Interview erfasst; Depressivität mit dem Beck-DepressionsInventar (BDI). Ergebnisse: Sexuell traumatisierte PTB-Patientinnen haben ein 4-fach höheres Risiko, Beeinträchtigungen der sexuellen Funktionsfähigkeit zu erleben als PTB-Patientinnen mit nichtsexuellem Trauma. Bei Vorliegen einer depressiven Störung zusätzlich zur PTB steigt das Risiko für sexuelle Dysfunktionen um das 3-fache gegenüber PTB-Patientinnen ohne komorbide Depression. Mit der sexuellen Zufriedenheit hängt die Art des Traumas hingegen nicht zusammen. Während drei Viertel der PTB- und Depressionspatientinnen sexuelle Dysfunktionen berichten, gilt dies nur für jede zweite Angstpatientin (außer PTB). Diskussion: Sowohl die Art des Traumas als auch komorbide Depressionen stehen im Zusammenhang mit sexuellen Dysfunktionen bei PTB-Patientinnen. Insbesondere bei Patientengruppen mit einem erhöhten Risiko für Beeinträchtigungen der sexuellen Funktionen ist es wichtig, die Thematik bei therapeutischen Interventionen zu berücksichtigen.

\section{Key Words}

Posttraumatic stress disorder . Sexual dysfunctions . Sexual satisfaction $\cdot$ Sexuality

\section{Summary}

Sexual Dysfunctions and Sexual Satisfaction in Female PTSD Patients

Background: Sexual dysfunctions are a common problem in female patients with posttraumatic stress disorder (PTSD). However, only few studies have investigated the incidence of sexual dysfunctions in PTSD patients accounting for the type of traumatisation (sexual vs non-sexual), the role of co-morbid depression as well as the comparison to other psychiatric disorders (clinical specification). Patients and Methods: In this study, 351 female outpatients were tested with a standardised clinical diagnostic instrument and assigned to the following 3 groups: patients with PTSD ( $n=89$ ), with other anxiety disorders ( $n=$ $157)$ and with depressive disorders $(n=105)$. Information about aspects of sexual satisfaction und sexual functioning was assessed by a short questionnaire ('Kurzfragebogen für Sexualität', KFS). The type of trauma was assessed by interview, and the level of depressive symptoms with the Beck Depression Inventory (BDI). Results: The risk of suffering from sexual dysfunctions was 4 times higher for sexually compared to nonsexually traumatised patients, and 3 times higher in the case of co-morbid depression compared to PTSD patients without co-morbid depression. Yet, there was no association between sexual satisfaction and type of trauma. Only half of the patients with anxiety disorder (except PTSD) suffer from sexual dysfunctions compared to 3 out of 4 patients with depression or PTSD. Discussion: Both the type of trauma and co-morbid depression are related to sexual dysfunction in patients with PTSD. Especially with patient groups that hold an increased risk of developing sexual dysfunctions, it is important to consider this topic during therapeutic interventions.

\section{KARGER}

Fax +497614520714

Information@Karger.de

www.karger.com (c) 2009 S. Karger GmbH, Freiburg

Accessible online at:

www.karger.com/ver 


\section{Hintergrund}

Sexuelle Probleme werden von der Mehrzahl der Patientinnen mit Traumaerfahrung oder mit der Diagnose einer posttraumatischen Belastungsstörung (PTB) berichtet [z.B. Becker et al., 1986; Kotler et al., 2000]. Übersichtsarbeiten zeigen, dass Opfer sexueller Traumatisierung im Kindes- und Jugendalter als Erwachsene vermehrt sexuelle Dysfunktionen aufweisen [Beitchman et al., 1992; Leonard und Folette, 2002; Najman et al., 2005]. Die Prävalenz sexueller Beeinträchtigungen erreicht in dieser Gruppe Werte bis $>80 \%$. Dies gilt vor allem für Probleme in frühen Phasen des sexuellen Reaktionszyklus (Appetenz-, Erregungsschwierigkeiten) und Phobien hinsichtlich sexueller Aktivität. Bei den meisten Vergewaltigungsopfern ist darüber hinaus die sexuelle Zufriedenheit eingeschränkt [Davis und Petretic-Jackson, 2000; Leonard und Follette, 2002].

Erklärungen für diese Befunde sind umstritten. Einerseits ist denkbar, dass unabhängig von der Ausbildung einer PTB, die Art der Traumatisierung (sexuell vs. nichtsexuell) eine Rolle bei der Entstehung sexueller Dysfunktionen spielt. Konditionierungstheoretische Überlegungen bieten in diesem Fall plausible Erklärungsmodelle, z.B. für die Ausbildung spezifischer Ängste und sexueller Funktionsstörungen oder für die negative Bewertung sexueller Stimuli im Rahmen einer PTB nach sexueller Traumatisierung [z.B. Becker et al., 1986; de Silva, 1999]. Nur sehr wenige Studien haben allerdings bisher die Beziehung zwischen sexuellen Dysfunktionen und der Art der Traumatisierung systematisch betrachtet. So zeigt die Arbeit von Tagay et al. [2004], dass PTB-Patienten, deren Trauma durch Menschen (körperliche oder sexuelle Gewalt) verursacht wurde, häufiger sexuelle Dysfunktionen erleben, als PTB-Patienten, die einem zufälligen Trauma oder einer Katastrophe ausgesetzt waren.

Darüber hinaus ist denkbar, dass - unabhängig vom Traumatyp - die Symptome der PTB die Entwicklung von sexuellen Dysfunktionen erklären. Die im DSM-IV [Saß et al., 1998] in Cluster C der PTB aufgeführten Vermeidungssymptome umfassen unter anderem emotionale Taubheit sowie Gefühle der Entfremdung von anderen, sodass indirekte Auswirkungen auf die Sexualität gut vorstellbar sind [Frewen et al., 2008]. Der Zusammenhang zwischen nichtsexuellen Traumatisierungen und der sexuellen Funktionsfähigkeit im Erwachsenenalter wurde allerdings erst vereinzelt erforscht. Die Ergebnisse dazu beschränken sich zudem zumeist auf männliche Patienten, deren PTB sich infolge von Kriegstraumatisierungen entwickelte. So zeigte sich, dass mehr als die Hälfte der männlichen Kriegsveteranen mit einer PTB unter Erektionsstörungen, Ejaculatio praecox oder verminderter sexueller Appetenz litt. Beeinträchtigungen der sexuellen Zufriedenheit traten bei ca. einem Drittel der befragten Männer auf [Letourneau et al., 1997; Kotler et al., 2000]. Auch bei Unfallopfern mit PTB wurden negative Auswirkungen auf die sexuelle Funktionsfähigkeit beschrieben [de Silva, 1999]. An- dere traumatische Ereignisse wie der Verlust eines Säuglings können das Sexualleben der zurückbleibenden Eltern ebenfalls beeinträchtigen, z.B. in Form von sexueller Aversion bei der Frau [Berry, 1999].

Sexuelle Funktionsfähigkeit und Zufriedenheit werden unabhängig von Traumatyp und PTB-Diagnose durch zahlreiche weitere Faktoren beeinflusst [Kockott, 1988]. Insbesondere (komorbide) depressive Störungen gehen mit sexuellen Dysfunktionen einher [z.B. Bonierbale et al., 2003; Michael und O'Keane, 2000;], vor allem mit dem Verlust bzw. der Minderung der sexuellen Appetenz (75\% der depressiven Patienten; Michael und O'Keane [2000]). Aber auch die sexuelle Erregungs- oder die Orgasmusfähigkeit kann eingeschränkt sein [Kennedy et al., 1999]. Weniger Daten liegen zur sexuellen Funktionsfähigkeit bei Patienten mit Angststörungen vor. Ergebnisse deuten darauf hin, dass auch bei Panik- oder Zwangsstörungen das sexuelle Interesse verringert ist; jedoch wurde eine depressive Komorbidität nicht immer kontrolliert [van Minnen und Kampmann, 2000; Rasmussen und Tsuang, 1986]. Ergebnisse aus der Grundlagenforschung [vgl. Barlow, 2002] belegen allerdings bei Gesunden einen positiven Zusammenhang zwischen Angsterleben und sexueller Funktionsfähigkeit.

Insgesamt lässt sich der Forschungsstand wie folgt zusammenfassen: Sexuelle Dysfunktionen sind im Zusammenhang mit einer PTB häufiger. Studien zur klinischen Spezifität, die belegen können, dass die Art des im Vordergrund der PTB stehenden «Index»-Traumas (sexuell vs. nichtsexuell) mit der Störungsrate im sexuellen Bereich assoziiert ist, fehlen genauso wie Studien, die zeigen, dass sexuelle Funktions-/Zufriedenheitseinschränkungen bei PTB-Patientinnen tatsächlich häufiger sind als bei Patientinnen mit anderen Störungen wie z.B. anderen Angststörungen oder Depressionen. Zudem steht trotz der hohen Komorbidität von depressiven Störungen und PTB [Kessler et al., 1995; Perkonigg et al., 2000] und des engen Zusammenhangs zwischen depressiven Störungen und sexuellen Problemen [z.B. Michael und O'Keane, 2000] eine kombinierte Untersuchung dieser Einflussfaktoren (Art des Traumas, depressive Störung) auf die Häufigkeit sexueller Dysfunktionen bei PTB-Patientinnen aus. Die vorliegende Studie stellt einen ersten Schritt zur Klärung dieser komplexen Fragestellung dar. Dabei beschränken wir uns auf weibliche Patienten.

Im Einzelnen wurden die folgenden Fragestellungen und Hypothesen untersucht: (1) Besteht bei PTB-Patientinnen ein Zusammenhang zwischen der Art des Index-Traumas (sexuell vs. nichtsexuell) und sexuellen Dysfunktionen bzw. Beeinträchtigungen der sexuellen Zufriedenheit unabhängig von komorbiden depressiven Störungen? Trotz eines erwarteten, substanziellen Beitrags komorbider Depression zur Erklärung sexueller Funktions- und Zufriedenheitsbeeinträchtigungen wird ein davon unabhängiger Einfluss der Art des Traumas dahingehend angenommen, dass bei einem sexuellen IndexTrauma häufiger sexuelle Funktionsbeeinträchtigungen vor- 
liegen und die sexuelle Zufriedenheit häufiger eingeschränkt ist als bei einem nichtsexuellen Index-Trauma. (2) Besteht ein Zusammenhang zwischen der Art der klinischen Störung (PTB, andere Angststörungen, Depression) und sexuellen Dysfunktionen/Einschränkungen der sexuellen Zufriedenheit? Es wird erwartet, dass PTB-Patientinnen häufiger von Einschränkungen der sexuellen Funktionsfähigkeit/Zufriedenheit betroffen sind als Patientinnen mit anderen Angststörungen, jedoch nicht stärker als Depressionspatientinnen.

\section{Patientinnen und Methoden}

\section{Stichprobe}

Diagnosen: Es wurden 351 Psychotherapiepatientinnen untersucht, die sich von Januar 2003 bis April 2006 in der Institutsambulanz und Tagesklinik für Psychotherapie der Technischen Universität Dresden zur Verhaltenstherapie angemeldet hatten und eine Diagnose in den Bereichen PTB, Angst oder depressive Störungen aufwiesen. Die Diagnosestellung erfolgte nach DSM-IV [Saß et al, 1998] mittels standardisiertem Interview [Münchener Composite International Diagnostic Interview, M-CIDI; Wittchen und Pfister, 1997]. Ausgeschlossen wurden Patientinnen, bei denen die Diagnose einer bipolaren, einer psychotischen Störung oder sowohl einer Angst- (nicht PTB) als auch einer depressiven Störung vorlag. 89 Patientinnen erfüllten die Diagnose einer PTB, 157 Patientinnen litten an mindestens einer anderen Angststörung ohne komorbide Depression (Panikstörung mit/ohne Agoraphobie, soziale Phobie spezifische Phobie, generalisierte Angststörung, Zwangsstörung), und 105 Patientinnen erfüllten die Kriterien für eine depressive Störung (Major Depression, Dysthymie) ohne komorbide Angststörung. Die soziodemographischen und psychometrischen Kennwerte der drei Gruppen sind Tabelle 1 zu entnehmen. Bei $62.9 \%$ der PTB-Patientinnen lag auch eine depressive Störung, bei $51.7 \%$ mindestens eine weitere Angststörung vor. Die Gruppen der PTB-, Angst- und Depressionspatientinnen waren vergleichbar hinsichtlich Alter und Partnerschaftsstatus, nicht jedoch im Bildungsniveau und im Grad an Depressivität: PTB-Patientinnen hatten höhere Depressivitätswerte als Angst- (Bonferroni adjust. $\mathrm{p} \leq 0,001$ ) oder Depressionspatientinnen (Bonferroni adjust. $\mathrm{p} \leq 0,05$ ). Die Gruppen PTB, Angst und Depression wiesen hingegen hinsichtlich der Selbstauskunft, ob sie derzeit Psychopharmaka einnähmen, keine signifikanten Differenzen auf.

Art des Traums: Die PTB-Patientinnen wurde nach der Art des berichteten Index-Traumas aufgeteilt: eine sexuelle Index-Traumatisierung (Vergewaltigung, sexueller Missbrauch, sexuelle Nötigung/sexualisierte Gewalt) lag bei 40 Patientinnen vor; 49 hatten ein nichtsexuelles IndexTrauma erlebt (körperliche Bedrohung, Angriffe, Überfälle oder nichtsexuelle Gewalt, Naturkatastrophen, Unfälle, Konfrontation mit Sterben oder Tod einer Person). Die PTB-Subgruppen sind in Hinblick auf Alter, Bildungsniveau, Partnerschaftsstatus und Einnahme von Psychopharmaka vergleichbar. Auch komorbide Depressionen lagen in beiden PTBSubgruppen vergleichbar oft vor (sexuelles Index-Trauma: 60.0\%, nichtsexuelles Index-Trauma: $\left.65.3 \% ; \chi_{(1)}^{2}=0,27, p=0,61\right)$. Patientinnen beider PTB-Subgruppen erlebten durchschnittlich drei verschiedene Arten von Traumata (sexuelles Index-Trauma: $\mathrm{M}=2,98, \mathrm{SD}=1,31$; nichtsexuelles Index-Trauma: $\mathrm{M}=2,90, \mathrm{SD}=1,20)$ und unterschieden sich somit diesbezüglich nicht signifikant $(T=0,290, p=0,773)$.

\section{Messinstrumente}

Kurzfragebogen zur Sexualität: Alle Patientinnen bearbeiteten routinemäßig im Rahmen der Eingangsuntersuchung vor Beginn der Therapie den Kurzfragebogen für Sexualität (KFS), die deutsche Version des Massachuetts General Hospital Sexual Functioning Questionnaire [MGH; Labbate und Lare, 2001; vgl. Reinecke et al., 2006]. Der KFS ist ein wenig zeitintensives Screeninginstrument zur Erfassung sexueller Dysfunktionen und sexueller Zufriedenheit. Mithilfe 5 kurzer Fragen wird durch Selbstauskunft der Patientinnen das Ausmaß ihrer sexuellen Funktionsfähigkeit hinsichtlich Appetenz, Erregungsfähigkeit, Orgasmus- und Lubrikationsfähigkeit sowie sexueller Zufriedenheit erfragt. Die Patientinnen wurden gebeten, ihre sexuelle Funktionsfähigkeit und Zufriedenheit bezogen auf den Zeitraum des vergangenen Monats jeweils anhand einer 6-stufigen Ratingskala einzuschätzen. Der KFS besitzt trotz seiner Kürze eine zufriedenstellende konvergente Validität zum Strukturierten Interview Sexueller Funktionsstörungen [SISEX; Reitz et al., 2005], einem Instrument zur Diagnostik sexueller Funktionsstörungen nach DSM-IV.

Beck-Depressions-Inventar: Das Selbstbeurteilungsverfahren [BDI; dt. Version: Hautzinger et al., 1994] erfasst den Schweregrad depressiver Symptome in der vergangenen Woche.

\section{Auswertung und Statistik}

Der Auswertung der Angaben zur sexuellen Funktionsfähigkeit bzw. Zufriedenheit (KFS) wurde in Anlehnung an Reinecke et al. [2006, p. 169] zugrunde gelegt, dass Werte $\leq 3$ (d.h. die Wahl der Kategorien «größer als sonst», «normal» und «minimal geringer») als «normale Schwankungen sexuellen Interesses und sexueller Funktionen» aufgefasst werden. Werte $\geq 4$ (d.h. die Wahl der Kategorien «etwas geringer», «deutlich geringer» und «nicht vorhanden») wurden als Hinweis auf Beeinträchtigungen der sexuellen Funktionsfähigkeit bzw. Zufriedenheit interpretiert. Der Zusammenhang zwischen der Art des Index-Traumas (sexuell vs. nichtsexuell) und sexuellen Dysfunktionen in mindestens einem der erfragten Bereiche der sexuellen Funktionsfähigkeit (sexDys $\geq 1$ ) bzw. Beeinträchtigungen der sexuellen Zufriedenheit (Ja vs. Nein) wurden unter Berücksichtigung des Einflusses komorbider Depressionen mittels multipler logistischer Regressionsanalysen untersucht. Ergänzend wurde eine ANOVA zur Feststellung von Unterschieden in der Bandbreite sexueller Einschränkungen (Anzahl der betroffenen Bereiche) in Abhängigkeit von der Art des Index-Traumas (Depressionsdiagnose als Kovariate) durchgeführt. Zur Erfassung des Zusammenhangs zwischen den unterschiedlichen klinischen Störungsbildern (PTB, Angst, Depression) wurden ebenfalls logistische Regressionen herangezogen. Alle statistischen Analysen wurden mit SPSS Version 16 durchgeführt.

\section{Ergebnisse}

\section{Sexuelle Dysfunktionen und Art des Index-Taumas}

In Tabelle 2 sind die relativen Häufigkeiten von sexuellen Dysfunktionen und Beeinträchtigungen der sexuellen Zufriedenheit bei PTB-Patientinnen in Abhängigkeit von der Art des Index-Traumas dargestellt. Daraus geht hervor, dass mindestens 4 von 5 PTB-Patientinnen mit einem sexuellen IndexTrauma sexuelle Dysfunktionen in mindestens einem der vier erfassten Bereiche der sexuellen Funktionsfähigkeit (sexuelles Interesse, sexuelle Erregungsfähigkeit, Orgasmusfähigkeit, Lubrikationsfähigkeit; sexDys $\geq 1$ ) berichteten; ebenso wie 3 von 5 der PTB-Patientinnen mit einem nichtsexuellen IndexTrauma. Es zeigte sich, dass PTB-Patientinnen mit sexuellem Index-Trauma unter Berücksichtigung komorbider depressiver Erkrankungen im Vergleich zu PTB-Patientinnen mit nichtsexuellem Index-Trauma ein 3,8-fach erhöhtes Risiko haben, sexuelle Dysfunktionen zu entwickeln. Das Vorliegen einer depressiven Erkrankung erhöhte das Risiko für sexuelle Dysfunktionen um das 3,1-fache. Insofern liegen mit der Art des Index-Traumas und komorbiden Depressionen zwei be- 
Tab. 1. Soziodemographische und psychometrische Charakteristika der Angst-, Depressions- und PTB-Patientinnen (inkl. Differenzierung nach Art Index-Traumas) (Angaben in \%, außer Alter)

\begin{tabular}{|c|c|c|c|c|c|c|c|c|c|}
\hline & $\begin{array}{l}\text { Angst } \\
(\mathrm{n}=157)\end{array}$ & $\begin{array}{l}\text { Depression } \\
(\mathrm{n}=105)\end{array}$ & $\begin{array}{l}\text { PTB } \\
(\mathrm{n}=89)\end{array}$ & Prüfgröße & $\mathrm{p}$ & $\begin{array}{l}\text { PTB SEX } \\
(\mathrm{n}=40)\end{array}$ & $\begin{array}{l}\text { PTB NONSEX } \\
(\mathrm{n}=49)\end{array}$ & Prüfgröße & $\mathrm{p}$ \\
\hline \multicolumn{10}{|l|}{ Alter, Jahre } \\
\hline Mittelwert & 37,5 & 36,5 & 33,8 & $\mathrm{~F}=2,11$ & n.s. & 32,3 & 35,0 & $\mathrm{~T}=1,07$ & n.s. \\
\hline SD & 13,8 & 14,0 & 11,6 & & & 11,8 & 11,5 & & \\
\hline Range & $17-78$ & $16-69$ & $16-62$ & & & $16-62$ & $18-61$ & & \\
\hline Schulabschluss & & & & $\chi^{2}=14,25$ & $\leq 0,05$ & & & $\chi^{2}=2,78$ & n.s. \\
\hline 9. Klasse & 9,6 & 6,7 & 16,9 & & & 20,0 & 14,3 & & \\
\hline 10. Klasse & 41,4 & 39,0 & 47,2 & & & 47,5 & 46,9 & & \\
\hline Abitur/Hochschule & 38,2 & 45,7 & 22,5 & & & 17,5 & 26,6 & & \\
\hline Kein Abschluss/keine Angaben & 10,8 & 8,6 & 13,4 & & & 15,0 & 12,2 & & \\
\hline Bestehende Partnerschaft & 79,6 & 69,4 & 69,7 & $\chi^{2}=6,27$ & n.s. & 72,5 & 63,7 & $\chi^{2}=0,278$ & n.s. \\
\hline Psychopharmaka & 35,7 & 39,0 & 44,9 & $\chi^{2}=2,05$ & n.s. & 45,0 & 44,9 & $\chi^{2}=0,000$ & n.s. \\
\hline Depressive Symptomatik (BDI) & 11,4 & 17,9 & 21,2 & $\mathrm{~F}=38,08$ & $\leq 0,001$ & 22,8 & 19,8 & $\mathrm{~T}=1,24$ & n.s. \\
\hline
\end{tabular}

Tab. 2. Multiple logistische Regressionsanalysen mit den Prädiktoren Art des Index-Traumas und komorbide Depression für das Vorliegen von sexuellen Dysfunktionen bzw. Beeinträchtigungen der sexuellen Zufrie denheit bei PTB-Patientinnen

\begin{tabular}{|c|c|c|c|c|c|c|}
\hline & \multirow{2}{*}{$\begin{array}{l}\text { SEX } \\
(n=40) \\
\%\end{array}$} & \multirow{2}{*}{$\begin{array}{l}\text { NONSEX } \\
(\mathrm{n}=49) \\
\%\end{array}$} & \multicolumn{2}{|c|}{ SEX vs. NONSEX } & \multicolumn{2}{|c|}{$\begin{array}{l}\text { Komorb. Depression } \\
\text { Ja vs. Nein }\end{array}$} \\
\hline & & & $\overline{\mathrm{OR}}$ & $95 \% \mathrm{CI}$ & $\overline{\mathrm{OR}}$ & $95 \% \mathrm{CI}$ \\
\hline SexDys $\geq 1$ & 85,0 & 63,3 & $3,77^{*}$ & $1,27-11,21$ & $3,10 *$ & $1,12-8,57$ \\
\hline Sexuelles Interesse & 70,0 & 57,1 & 1,98 & $0,78-5,03$ & $3,52 * *$ & $1,39-8,90$ \\
\hline Sexuelle Erregungsfähigkeit & 75,0 & 55,1 & $2,81 *$ & $1,08-7,35$ & $3,29 *$ & $1,28-8,46$ \\
\hline Orgasmusfähigkeit & 77,5 & 55,3 & $3,13 *$ & $1,18-8,32$ & $2,70 *$ & $1,03-7,06$ \\
\hline Lubrikationsfähigkeit & 43,2 & 36,2 & 1,45 & $0,59-3,61$ & $2,65^{*}$ & $1,00-7,05$ \\
\hline Beeinträchtigung sex. Zufriedenheit & 63,2 & 59,6 & 1,35 & $0,53-3,46$ & $4,21 * *$ & $1,64-10,81$ \\
\hline
\end{tabular}

sexDys $\geq 1$ : Einer oder mehrere Bereiche der sexuellen Funktionsfähigkeit sind durch sexuelle Dysfunktionen betroffen. SEX $=$ Sexuelles Index-Trauma; NONSEX = nichtsexuelles Index-Trauma. $* \mathrm{p} \leq 0,05 ; * \mathrm{p} \leq 0,01$. deutsame und vergleichbar starke Einflussfaktoren auf das Auftreten sexueller Dysfunktionen bei PTB-Patientinnen vor. Das Spektrum sexueller Dysfunktionen, d.h. die Anzahl der erfragten vier Bereiche der sexuellen Funktionsfähigkeit, die von sexuellen Dysfunktionen betroffen sind, umfasste bei PTB-Patientinnen mit nichtsexuellem Index-Trauma 2,0, während PTB-Patientinnen mit sexuellem Index-Trauma durchschnittlich in 2,6 Bereichen eine Reduktion der sexuellen Funktionsfähigkeit angaben. Auch bei Kontrolle des Einflussfaktors «komorbide Depression» konnte ein signifikanter Gruppenunterschied nachgewiesen werden $(F=4,91, p \leq 0,05)$, der eine größere Bandbreite von Beeinträchtigungen bei PTB-Patientinnen mit sexuellem Index-Trauma aufzeigt. Zwischen der Art des Index-Traumas und Beeinträchtigungen der sexuellen Zufriedenheit besteht hingegen kein signifikanter Zusammenhang. Allerdings kommt komorbiden depressiven Erkrankungen auch hier Bedeutung zu. So ist das Risiko von PTB-Patientinnen mit komorbider Depression, Beeinträchtigungen der sexuellen Zufriedenheit zu erleben, um das 4,2fache erhöht.

\section{Vergleich von PTB mit Angst- und Depression}

Drei Viertel der PTB- und der Depressionspatientinnen berichteten sexuelle Dysfunktionen in mindestens einem sexuellen Funktionsbereich (sexDys $\geq 1$; Tab. 3), dies gilt jedoch nur für jede zweite Angstpatientin. Sowohl für die Gesamtgruppe der PTB-Patientinnen als auch für die Subgruppe mit sexuellem Index-Trauma ist im Gegensatz zu Patientinnen mit anderen Angststörungen das Risiko für das Auftreten von sexuellen Dysfunktionen (um das 2,7- bzw. sogar 5,6fache) und für Einschränkungen der sexuellen Zufriedenheit (um das 2,3-bzw. 2,5-fache) deutlich höher. Allerdings zeigte sich für PTB-Patientinnen weder für das Auftreten sexueller Dysfunktionen (sexDys $\geq 1$ ) noch für Beeinträchtigungen der sexuellen Zufriedenheit ein erhöhtes Risiko im Vergleich zu Patientinnen mit der Hauptdiagnose Depression. Im Gegensatz dazu ist das Risiko für die PTB-Subgruppe mit nichtsexuellem Index-Trauma hinsichtlich des Auftretens sexueller Dysfunktionen (sexDys $\geq 1$ ) nicht signifikant größer als bei anderen Angstpatientinnen. Analog zu den PTB-Patientinnen mit sexuellem Index-Trauma zeigte sich auch hier kein er- 


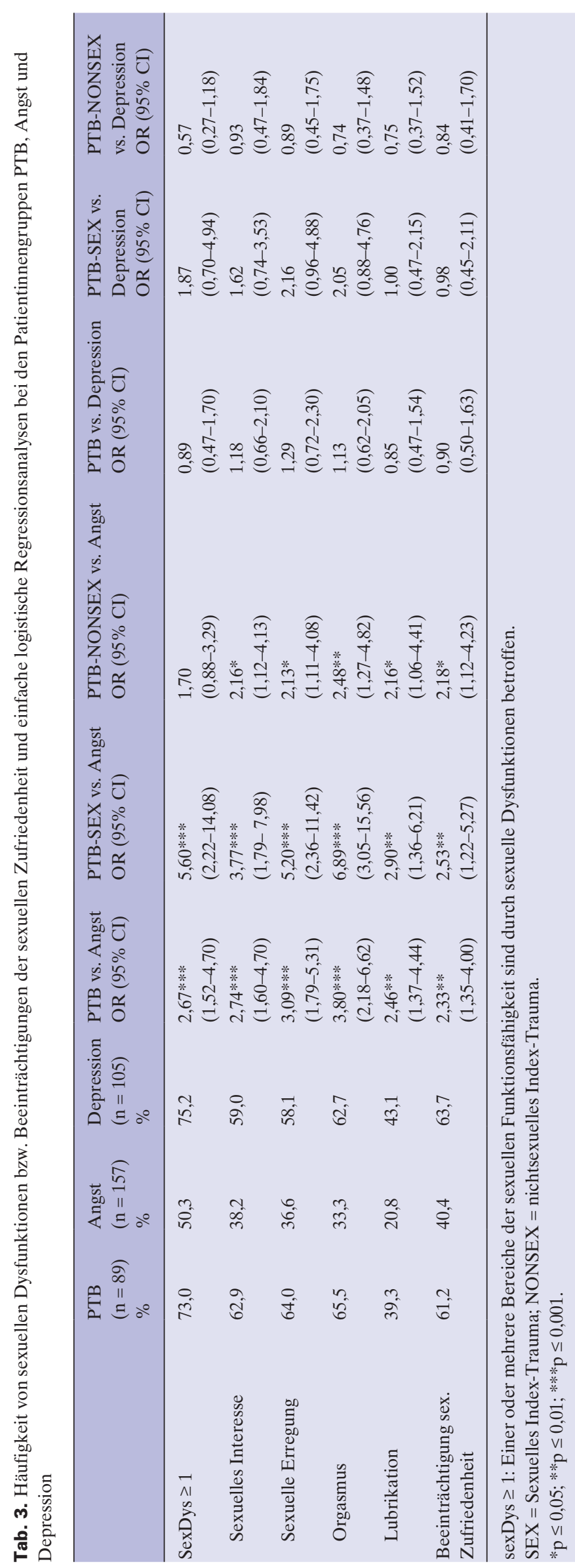

höhtes Risiko für das Auftreten sexueller Dysfunktionen und Einschränkungen der sexuellen Zufriedenheit gegenüber Patientinnen mit der Hauptdiagnose Depression.

\section{Diskussion}

Die Ergebnisse der vorliegenden Studie zeigen: Sexuelle Funktionsbeeinträchtigungen und Einschränkungen der sexuellen Zufriedenheit wurden in unserer Stichprobe ambulanter Psychotherapiepatientinnen grundsätzlich häufig genannt; mindestens jede zweite Patientin gibt sexuelle Dysfunktionen in einem oder mehreren der Bereiche sexuelles Interesse, sexuelle Erregungsfähigkeit, Orgasmus- oder Lubrikationsfähigkeit an. Diese waren allerdings besonders akzentuiert, wenn bei Patientinnen mit PTB ein sexuelles Index-Trauma oder aber eine depressive Erkrankung (unabhängig von der Diagnose einer PTB) vorlag. Zudem führte auch eine komorbide Depressionsdiagnose bei PTB-Patientinnen zu einem erhöhten Risiko für sexuelle Dysfunktionen und Beeinträchtigungen der sexuellen Zufriedenheit.

Die erhöhte Wahrscheinlichkeit für sexuelle Funktionsbeeinträchtigungen in Folge einer sexuellen im Vergleich zu einer nichtsexuellen Index-Traumatisierung bei PTB wurde somit in dieser Studie unter Kontrolle komorbider Depressionen bestätigt. So ist das Risiko, Einschränkungen der sexuellen Funktionsfähigkeit zu erleben, bei Patientinnen, deren PTB sich infolge eines sexuellen Traumas entwickelte, fast 4-mal größer als bei Patientinnen, deren für die PTB ausschlaggebendes Trauma nichtsexueller Natur ist. Sowohl lerntheoretische Ansätze als auch kognitive Modelle der PTB [z.B. Ehlers und Clark, 2000] legen nahe, dass Auslösereize, die mit dem Trauma assoziiert werden, und dysfunktionale Bewertungen, die mit dem Ereignis in Verbindung stehen, zu wiederkehrenden starken Emotionen führen können, die ihrerseits zusätzlich durch Vermeidungsverhalten aufrechterhalten werden. Durch das Fehlen einer Kontrollgruppe ohne PTB nach sexuellem Trauma bleibt allerdings offen, ob ein sexuelles Trauma per se oder erst seine pathologische Verarbeitung mit den sexuellen Schwierigkeiten zusammenhängt. Trotz dieser Einschränkungen lässt sich jedoch zusammenfassen, dass das Risiko, unter sexuellen Dysfunktionen zu leiden, steigt, wenn die Diagnose einer PTB nach sexuellem IndexTrauma vorliegt. Die sexuelle Zufriedenheit steht hingegen nicht in Zusammenhang mit der Art des Index-Traumas.

Der Faktor «Depressivität», der große Überlappungen mit dem Cluster der Vermeidung bei PTB aufweist, erwies sich in der vorliegenden Studie ebenfalls als entscheidend für die Auftretenswahrscheinlichkeit sexueller Dysfunktionen, und dieser (komorbide) Faktor ist bei PTB-Patientinnen im Vergleich zu Patientinnen mit anderen Angststörungen stärker ausgeprägt. Zudem zeigte sich, dass PTB-Patientinnen unabhängig von der Art des Index-Traumas nicht in größerem Ausmaß von sexuellen Funktions- und Zufriedenheitsbeein- 
trächtigungen betroffen sind als Patientinnen mit depressiven Störungen. Die Ergebnisse stehen im Einklang mit der bereits bekannten hohen Prävalenz sexueller Funktionsstörungen bei depressiven Patienten [z.B. Kennedy et al., 1999]. Der Faktor Depression scheint insbesondere bei der sexuellen Zufriedenheit eine Rolle zu spielen. Dies entspricht Befunden anderer Studien [z.B. Nicolosi et al., 2004].

Bei der Diskussion der Ergebnisse ist kritisch darauf hinzuweisen, dass diese aufgrund des Screeningcharakters des Erhebungsinstrumentes als vorläufig zu erachten sind. Für validere Aussagen wäre ein klinisches Interview nötig (z.B. das Strukturierte Interview Sexueller Funktionsstörungen, SISEX [Reitz et al., 2005]). Weiterhin fehlt eine nichtklinische Kontrollgruppe im Vergleich der Gruppen bezüglich sexueller Funktions- und Zufriedenheitseinschränkungen. Weitere Aspekte, wie der Einfluss des Bildungsniveaus [vgl. Laumann et al., 1999] oder eine Generalisierung der Befunde auf männliche traumatisierte Patienten, könnten in folgenden Studien ebenfalls interessante und bedeutsame Variablen darstellen. Zudem sollte die partnerschaftliche Zufriedenheit berücksichtigt werden, die insbesondere bei Frauen eng mit der sexuellen Funktionsfähigkeit/Zufriedenheit zusammenhängt [z.B. Rosen et al., 2008; Schönbucher, 2007]. Hingegen lässt der Partnerschaftsstatus allein vermutlich kaum Rückschlüsse auf die sexuelle Funktionsfähigkeit zu, denn Sexualität und sexuelle Zufriedenheit lassen sich nicht auf Zweierbeziehungen beschränken. Auch autoerotische oder außerpartnerschaftliche sexuelle Aktivitäten können darauf Einfluss nehmen.

Die berichteten Beeinträchtigungen der sexuellen Funktionsfähigkeit und der sexuellen Zufriedenheit lassen es wünschenswert erscheinen, Sexualität im ambulanten Setting speziell bei
Patientinnen mit PTB und/oder Depressionen sowie insbesondere bei PTB-Patientinnen mit sexuellem Index-Trauma zu thematisieren und gegebenenfalls gesondert darauf einzugehen [vgl. Reinecke et al., 2006]. Für die klinische Behandlung ergibt sich die wichtige Aufgabe, die Patientinnen über die Zusammenhänge zwischen Trauma, Depression und sexuellen Störungen aufzuklären. Während im Falle einer (primären) Depression die sexuellen Probleme symptomatischer Ausdruck der Störung sein könnten, ist im Falle sexueller Traumatisierung gegebenenfalls eine direkte Bearbeitung der mit dem Trauma in Verbindung stehenden dysfunktionalen Bewertungen und Strategien im Zusammenhang mit den aktuellen sexuellen Schwierigkeiten indiziert [Boos, 2005]. Dies bedeutet z.B. das Erlernen der Diskriminierung zwischen Reaktionen auf Intrusionen mit starkem Wiedererlebenscharakter und einer realen Gefahreneinschätzung im Zusammenhang mit sexueller Aktivität. Hoyer et al. [2009] konnten zwar zeigen, dass sich sexuelle Dysfunktionen bei diagnostisch heterogenen Patienten einer Verhaltenstherapie auch ohne spezifische Intervention deutlich verringerten. $\mathrm{Ob}$ sich ein solcher Effekt aber auch bei Patientinnen mit der Behandlungsdiagnose PTB und speziell nach einem sexuellen Trauma einstellt, ist allerdings noch unklar und drängt sich als zukünftige Forschungsfrage auf.

Sexuelle Funktionsfähigkeit und Zufriedenheit üben einen entscheidenden Einfluss auf die Lebensqualität aus; auch Psychotherapiepatienten räumen ihnen einen hohen Stellenwert ein [Götzmann et al., 2003]. Angesichts der hohen Prävalenz von sexuellen Dysfunktionen und Beeinträchtigungen der sexuellen Zufriedenheit bei ambulanten Psychotherapiepatientinnen sollte daher die Sensibilität der Behandelnden dafür erhöht werden.

\section{Literatur}

Barlow DH: Anxiety and Its Disorders, ed 2. New York, Guilford, 2002.

Becker JV, Skinner LJ, Abel GG, Cichon J: Level of postassault sexual functioning in rape and incest victims. Arch Sex Behav 1986;15:37-49.

Beitchman JH, Zucker KJ, Hood JE, da Costa GA, Akman D, Cassavia E: A review of the long-term effects of child sexual abuse. Child Abuse Negl 1992; 16:101-118.

Berry M: Grief and psychosexual disturbance following the death of a young baby: some issues for practioners. Sex Marital Ther 1999;14:27-42.

Bonierbale M, Lançon C, Tignol J: The ELIXIR study: evaluation of sexual dysfunction in 4,557 depressed patients in France. Curr Med Res Opin 2003;19: 114-124.

Boos A: Kognitive Verhaltenstherapie nach chronischer Traumatisierung. Göttingen, Hogrefe, 2005

Davis JL, Petretic-Jackson PA: The impact of child sexual abuse on adult interpersonal functioning: A review and synthesis of the empirical literature. Aggression Violent Behav 2000;5:291-328.

de Silva P: Sexual consequences of non-sexual trauma. Sex Marital Ther 1999;14:143-150.

Ehlers A, Clark DM: A cognitive model of posttraumatic stress disorder. Behav Res Ther 2000;38:319-45.
Frewen PA, Lanius RA, Dozois DJA., Neufeld RWJ, Pain C, Hopper JW, Densmore M, Stevens TK: Clinical and neural correlates of alexithymia in posttraumatic stress disorder. J Abnorm Psychol 2008; 117:171-81.

Götzmann L, Capek S, Dutta M, Klaghofer R, Fuchs W, Buddeberg C: Einstellung zur Sexualität vor Beginn einer antidepressiven Therapie - eine Pilotstudie. Schweiz Arch Neurol Psychiatr 2003;154:276-280.

Hautzinger M, Bailer M, Worall H, Keller F (Hrsg): Beck-Depressions-Inventar BDI, ed 2. Bern, Huber, 1994.

Hoyer J, Uhman S, Rambow J, Jacobi F: Reduction of sexual dysfunction: by-product of cognitive-behavioural therapy for psychological disorders? Sex Relationship Ther 2009;24:64-73.

Kennedy SH, Dickens SE, Eidfeld BS, Bagby RM: Sexual dysfunction before antidepressant therapy in major depression. J Affect Disord 1999;56:201-208.

Kessler RC, Sonnega A, Bromet E, Hughes M, Nelson CB: Posttraumatic stress disorder in the National Comorbidity Survey. Arch Gen Psychiatry 1995;52: 1048-1060.

Kockott G: Weibliche Sexualität. Stuttgart, Hippokrates, 1988.
Kotler M, Cohen H, Aizenberg D, Matar M, Loewenthal U, Kaplan Z, Miodownik H, Zemishlany $\mathrm{Z}$ : Sexual dysfunction in male posttraumatic stress disorder patients. Psychother Psychosom 2000;69: 309-315.

Labbate LA, Lare SB: Sexual dysfunction in male psychiatric outpatients: validity of the Massachusetts General Hospital Sexual Functioning Questionnaire. Psychother Psychosom 2001;70:221-225.

Laumann EO, Paik A, Rosen RC: Sexual dysfunctions in the United States: prevalence and predictors. JAMA 1999;281:537-544.

Leonard LM, Follette VM: Sexual functioning in women reporting a history of child sexual abuse: review of the empirical literature and clinical implications. Annu Rev Sex Res 2002;13:346-389.

Letourneau EJ, Schewe PA, Frueh BC: Preliminary evaluation of sexual problems in combat veterans with PTSD. J Trauma Stress 1997;10:125-132.

Michael A, O'Keane V: Sexual dysfunction in depression. Hum Psychopharmacol 2000;15:337-345.

Najman JM, Dunne MP, Purdie DM, Boyle FM, Coxeter PD: Sexual abuse in childhood and sexual dysfunction in adulthood: an Australian population-based study. Arch Sex Behav 2005;34:517-526. 
Nicolosi A, Moreira ED Jr, Villa M, Glasser DB: A population study of the association between sexual function, sexual satisfaction and depressive symptoms in men. J Affect Disord 2004;82:235-243.

Perkonigg A, Kessler RC, Storz S, Wittchen H-U: Traumatic events and post-traumatic stress disorder in the community: prevalence, risk factors and comorbidity. Acta Psychiatr Scand 2000;101:46-59.

Rasmussen SA, Tsuang MT: Clinical characteristics and family history in DSM-III obsessive-compulsive disorder. Am J Psychiatry 1986;14:317-322.

Reinecke A, Schöps D, Hoyer J: Sexuelle Dysfunktionen bei Patienten einer verhaltenstherapeutischen Hochschulambulanz: Häufigkeit, Erkennen und Behandlung. Verhaltenstherapie 2006;16:166-172.
Reitz D, Küpper B, Hoyer J: Strukturiertes Interview für sexuelle Funktionsstörungen - erste Ergebnisse. Psychother Psychosom Med Psychol 2005;55:147.

Rosen RC, Bachmann GA: Sexual well-being, happiness, and satisfaction in women: The case for a new conceptual paradigm. J Sex Marital Ther 2008;34: 291-297.

$\mathrm{Sa}$ H, Wittchen H-U, Zaudig M: Diagnostisches und Statistisches Manual Psychischer Störungen (DSMIV). Deutsche Bearbeitung und Einführung. Göttingen, Hogrefe, 1998

Schönbucher V: Sexuelle Zufriedenheit von Frauen: Psychosoziale Faktoren: Eine Übersicht. Z Sexualforsch 2007;20:21-41.
Tagay S, Herpertz S, Langkafel M, Senf W: Trauma, posttraumatische Belastungsstörung und Somatisierung. Psychother Psychosom Med Psychol 2004;54: 198-205.

van Minnen A, Kampman M: The interaction between anxiety and sexual functioning: a controlled study of sexual functioning in women with anxiety disorders. Sexual Relationship Ther 2000;15:47-57.

Wittchen H-U, Pfister H: DIA-X-Interviews: Manual für Screeningverfahren und Interview: PC - Programm zur Durchführung des Interviews (Längsund Querschnittsuntersuchung), Auswertungsprogramm. Frankfurt/M, Swets und Zeitlinger, 1997. 